\title{
Smoking and interstitial lung diseases
}

\author{
George A. Margaritopoulos ${ }^{1}$, Eirini Vasarmidi ${ }^{2}$, Joseph Jacob ${ }^{3}$, Athol U. Wells ${ }^{1}$ \\ and Katerina M. Antoniou ${ }^{2}$
}

\begin{abstract}
Affiliations: ${ }^{1}$ Interstitial Lung Disease Unit, Royal Brompton Hospital, London, UK. ${ }^{2}$ Dept of Thoracic Medicine and Laboratory of Molecular and Cellular Pneumonology, Interstitial Lung Disease Unit, University Hospital of Heraklion, Heraklion, Greece. ${ }^{3}$ Dept of Radiology, Royal Brompton Hospital, London, UK.
\end{abstract}

Correspondence: Katerina M. Antoniou, Molecular and Cellular Pneumonology Laboratory, Dept of Thoracic Medicine, Medical School, University of Crete, Heraklion, 71110 Crete, Greece. E-mail: kantonioulamed.uoc.gr

ABSTRACT For many years has been well known that smoking could cause lung damage. Chronic obstructive pulmonary disease and lung cancer have been the two most common smoking-related lung diseases. In the recent years, attention has also focused on the role of smoking in the development of interstitial lung diseases (ILDs). Indeed, there are three diseases, namely respiratory bronchiolitisassociated ILD, desquamative interstitial pneumonia and pulmonary Langerhans cell histiocytosis, that are currently considered aetiologically linked to smoking and a few others which are more likely to develop in smokers. Here, we aim to focus on the most recent findings regarding the role of smoking in the pathogenesis and clinical behaviour of ILDs.

0 @ERSpublications

Smoking is implicated in the pathogenesis and clinical behaviour of interstitial lung disease http://ow.ly/PYLcT

\section{Introduction}

Cigarette smoking is a very common and addictive habit and is well known for its harmful effects because of the high number of chemicals contained. The role of smoking in many human diseases, such as chronic obstructive pulmonary disease (COPD), lung cancer and atherosclerosis, has already been defined [1-3]. Interestingly, the link between cigarette smoking and interstitial lung diseases (ILDs) is not yet well established.

Recently, VASSALLO et al. [4] proposed four groups of smoking-related ILDs based on associations with cigarette smoking (table 1). The first group includes respiratory bronchiolitis-associated ILD (RB-ILD), desquamative interstitial pneumonia (DIP) and pulmonary Langerhans cell histiocytosis (PLCH), three chronic diffuse lung diseases that are strongly aetiologically linked to smoking (fig. 1) [5, 6]. The second group consists of two acute ILDs, acute eosinophilic pneumonia and pulmonary haemorrhage syndrome, in which smoking seems to have an important pathogenetic role, although to a lesser extent than in the first group. The third group contains idiopathic pulmonary fibrosis (IPF) and rheumatoid arthritis-related ILD (RA-ILD), diseases in which smoking is increased in prevalence. Finally, the fourth group includes sarcoidosis and hypersensitivity pneumonitis, two diseases that are actually less likely to develop in smokers [4]. The protective effect of smoking in these diseases may result from suppression of T-helper cell (Th)1 immunity by cigarette smoking, but at a price in terms of other forms of damage and functional impairment of the immune system, potentially leading to alternative lung diseases. Nonetheless, even in sarcoidosis it has been demonstrated that when smokers develop the disease the outcome is worse than in nonsmokers [7].

Plainly, identifying the exact role of smoking in ILDs is extremely important as understanding the relevant pathogenetic pathways may allow the development of new drugs targeting pathways activated by smoking as well as the inclusion of ILDs in the campaigns against smoking, as is already happening with great

Received: June 152015 | Accepted after revision: July 112015

Conflict of interest: None declared.

Provenance: Publication of this peer-reviewed article was sponsored by F. Hoffman-La Roche Ltd, Basel, Switzerland (principal sponsor, European Respiratory Review issue 137).

Copyright CERS 2015. ERR articles are open access and distributed under the terms of the Creative Commons Attribution Non-Commercial Licence 4.0. 
TABLE 1 Classification of smoking related-interstitial lung diseases (ILDs)

1) Chronic ILDs strongly associated with cigarette smoking

2) Acute ILDs related to smoking

3) ILDs that are more prevalent in smokers

4) ILDs that may be less prevalent in smokers
Respiratory bronchiolitis-associated ILD

Desquamative interstitial pneumonia

Adult pulmonary Langerhans cell histiocytosis

Acute eosinophilic pneumonia

Pulmonary haemorrhage syndromes

Idiopathic pulmonary fibrosis

Rheumatoid arthritis-associated ILD

Hypersensitivity pneumonitis

Sarcoidosis

Reproduced and modified from [4] with permission from the publisher.

success for non-ILDs including COPD and lung cancer. It is also important from a clinical point of view as recognition of a smoking-related phenotype would facilitate early diagnosis and treatment, especially with regard to IPF, the most common and progressive form of ILD.

\section{Is smoking related to the pathogenesis of ILDs?}

There has been a lot of progress regarding the understanding of the role of smoking in the pathogenesis of ILDs, but many pieces of the jigsaw are still missing. The lungs of smokers are continuously exposed to insults, such as the cytotoxic, mutagenic and proinflammatory substances contained in cigarettes, leading to an increasing risk of the development of lung disorders. Respiratory bronchiolitis, which is present histologically in all active smokers, is characterised by the accumulation of pigmented "smoker's macrophages" in respiratory bronchioles and neighbouring alveoli, containing granular, yellow-brown cytoplasmic pigments [8]. Therefore, respiratory bronchiolitis may be considered a "normal" physiological response to cigarette smoking unless it is sufficiently severe to have the features of a clinically overt ILD, based on clinical symptoms or lung function impairment $[4,9-13]$. Thus, the definition of a healthy smoker refers to an asymptomatic smoker with normal lung function values, even if respiratory bronchiolitis is present. Oxidative stress, increased epithelial apoptosis and deregulated immune responses represent well known smoking induced mechanisms [14-21]. It is known that cigarette smoking causes inflammatory cell recruitment, mostly macrophages, which have reduced apoptosis and increased survival. Lung macrophages are also functionally affected and recent evidence suggests an induction of the M2 phenotype upon smoking stimulation [22-25]. The M2 phenotype of macrophages is known to enhance resolution of inflammation and tissue remodelling.

\section{Osteopontin}

Prasse et al. [26] showed that osteopontin (OPN), a glycoprotein with cytokine properties, is significantly increased in patients with smoking-related ILDs (fig. 2). This glycoprotein is found in the extracellular matrix of bone and has chemotactic properties for macrophages, monocytes, dendritic cells and Langerhans cells. Interestingly, this study demonstrated evidence of chronic nicotine stimulation in macrophages of patients with DIP and PLCH. The authors also proposed that nicotine is able to promote increased OPN and granulocyte-macrophage colony-stimulating factor production by alveolar macrophages, suggesting an association between nicotine and the accumulation of immune cells in smoking related-ILDs [26]. Nicotine is known to affect many organs in the human body and to alter the normal function of cells that express nicotine receptors.

\section{Nicotine}

Nicotine has pleiotropic effects which seem to be concentration-dependent; it has been hypothesised that it enhances fibrogenesis through several mechanisms [27] including endothelial and epithelial damage, production of proinflammatory cytokines, and activation of transforming growth factor (TGF)- $\beta$, macrophages and fibroblasts. An in vivo study, by Roomans et al. [28], reported that nicotine alters the homeostasis of epithelial cells in the lung, enhances inflammatory reactions and increases eosinophil numbers in the trachea. In the mouse kidney, nicotine increases the production of TGF- $\beta$ and the expression of epithelial-mesenchymal transition markers [29]. While in human hepatic cells it also induces TGF- $\beta$ and collagen expression, promoting fibrogenesis [30]. In addition, TGF- $\beta$ release is induced by smoke exposure in rat tracheal explants, potentially promoting airway remodelling [31]. 

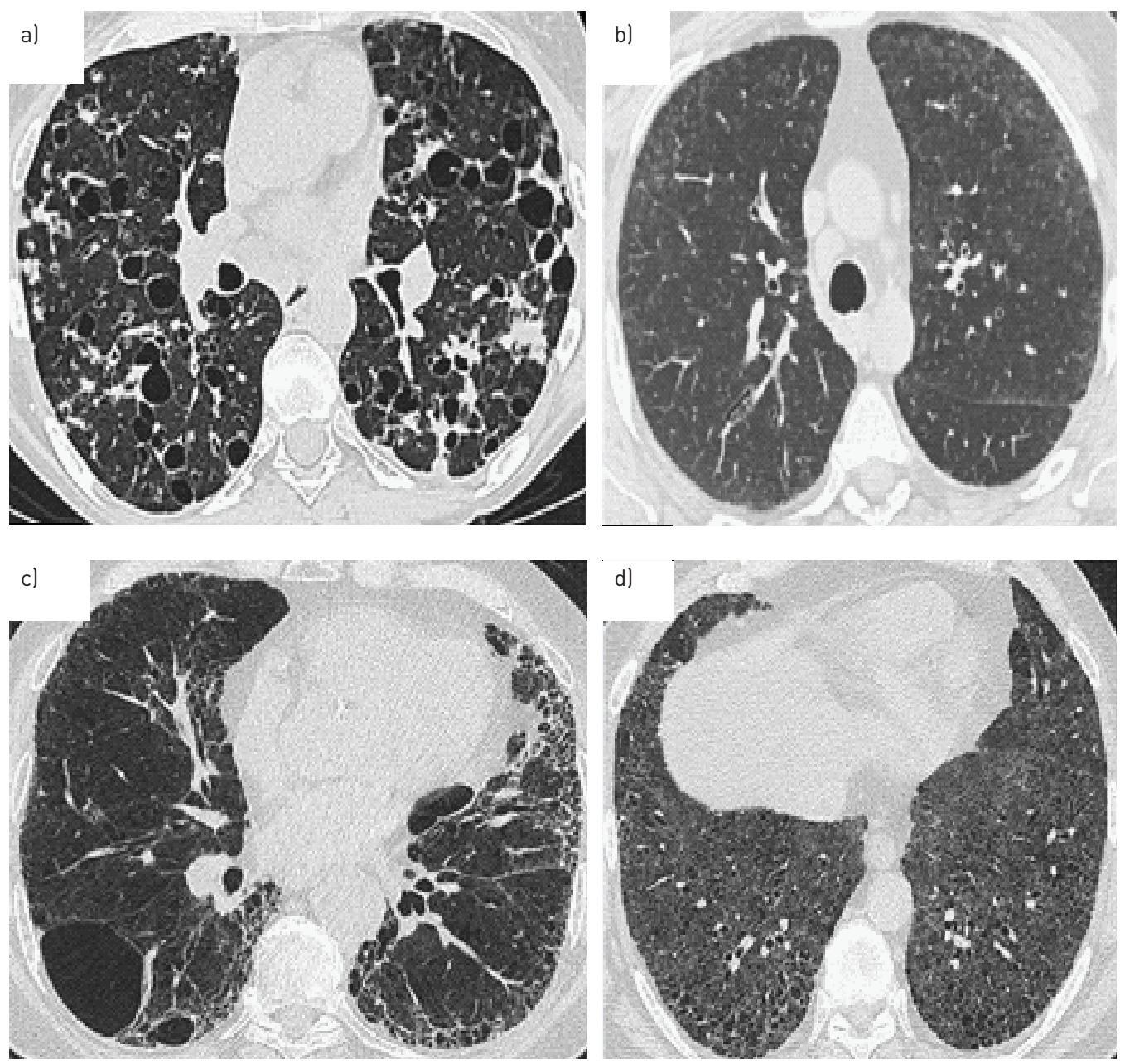

FIGURE 1 High-resolution computed tomography images of smoking-related interstitial lung diseases (ILDs). a) Pulmonary Langerhans cell histiocytosis, b) respiratory bronchiolitis-associated ILD, c) combined pulmonary fibrosis and emphysema, and d) desquamative interstitial pneumonia.

\section{Smoking-related epigenetic alterations}

Smoking promotes epigenetic alterations that persist for years even after smoking cessation. It has been detected that smokers display increased levels of promoter hypermethylation, and higher levels of DNA methyltransferase 1, resulting in decreased expression of several genes, mainly associated with the cell cycle [32]. Histone $\mathrm{H} 4$ acetylation, in parallel with reduced function of histone deacetylase-2, is also induced by smoking [33-35]. Two studies by Coward and colleagues suggested that defective histone acetylation and histone hypermethylation may have a role in the fibrogenic process in IPF [36, 37], highlighting the importance of epigenetic deregulation in the development of the disease.

\section{Citrullination}

Citrullination, a post-translational modification of proteins that alters their structure and has functional consequences regarding their immunogenicity, is triggered by smoking and appears to be characteristic of rheumatoid arthritis. Patients with anti-citrullinated protein antibodies (ACPAs) are more likely to have lung abnormalities [38,39]. Interestingly, FISCHER et al. [40] showed that, in some patients, ACPA positivity and lung abnormalities develop prior to joint disease. It is established that in smokers with rheumatoid arthritis there are citrullinated peptides in the bronchoalveolar lavage fluid cells, which are not present in nonsmokers [41]. Two recent studies revealed higher levels of specific ACPAs in RA-ILD patients than in patients with rheumatoid arthritis without $\operatorname{ILD}[42,43]$, and there was also an association with a usual interstitial pneumonia (UIP) pattern on high-resolution computed tomography (HRCT) [43]. These findings may be indicative of an important role for smoking and citrullination in the development of ILD related to rheumatoid arthritis and provide new diagnostic tools concerning lung involvement [38, 44]. 
FIGURE 2 Chronic nicotine stimulation leads to recruitment of inflammatory cells. nAchR: nicotinic acetylcholine receptors.

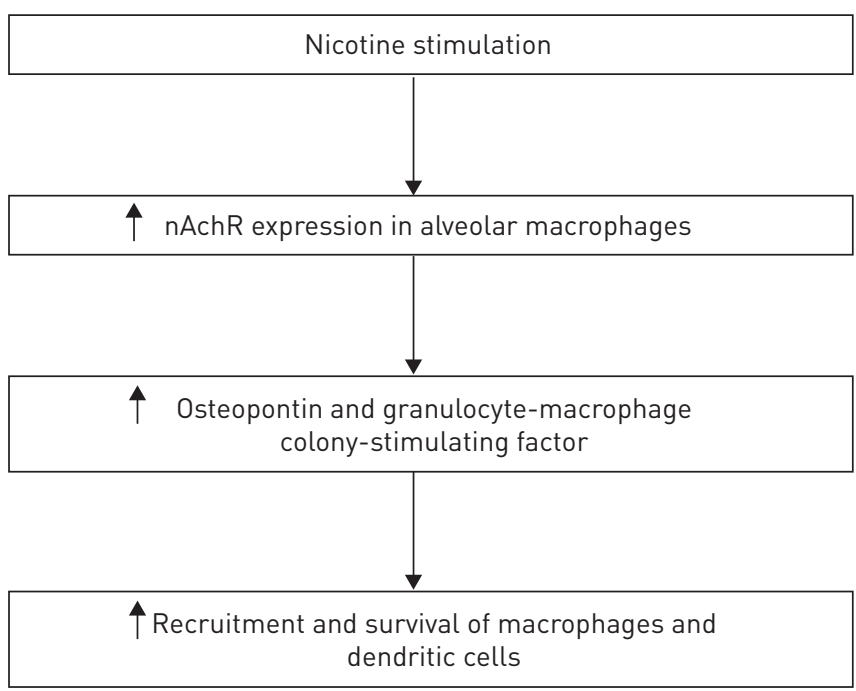

\section{Premature ageing}

Premature ageing may be an important pathogenetic mechanism in lung diseases [45-48]. Cellular senescence, telomere shortening, oxidative stress and DNA damage are considered hallmarks of the ageing process [49]. Telomeres are special nucleotide repeats at the ends of chromosomes that provide chromosomal stability. Telomeres shorten progressively during replication, without losing coding information. When telomeres reach a critical length, however, the cell dies. Cigarette smoking is known to promote telomere shortening and this phenomenon has been described in alveolar epithelial cells and in peripheral blood cells in IPF [50-52]. Interestingly, circulating leukocytes in smokers have shorter telomeres in a dose-dependent manner [53,54]. Additionally, DNA damage and oxidative stress have been found in higher levels in smokers [46, 55]. It has been demonstrated that tobacco, and other stimuli that cause DNA damage, result in an abnormal repair process and fibrosis, partially due to senescent bone marrow-derived stem cells [56]. These cells can migrate and make a major contribution to lung repair mechanisms. Cigarette smoke is also a known source of exogenous oxidants, resulting in an imbalance of pro-oxidant and antioxidant molecules, especially in the presence of a deficiency in the cellular control systems of reactive oxygen species (ROS) production $[45,57]$.

\section{Autophagy/mitophagy}

During ageing, proteins and cellular debris are ineffectively cleared and accumulate in the cell. Such a defect in cellular housekeeping mechanisms leads to senescence or apoptosis $[46,58,59]$. Autophagy is a tightly regulated and selective process responsible for the degradation of cellular components via the lysosomal pathway. It is vital for cellular homeostasis, as it represents a quality control mechanism for the cell and participates in bioenergetic adaptation to starvation. It has several physiological and pathophysiological functions contributing to human disease pathogenesis [60-63]. Mitophagy is the selective autophagy of mitochondria, which are central to healthy cell metabolism but can also inflict cellular damage and trigger apoptosis by releasing toxic by-products of oxidative phosphorylation. Oxidative stress causes mitochondrial depolarisation and dysfunction and the accumulation of impaired mitochondria determinates cell fate and function [64]. Mitophagy is the major mechanism for efficient clearance of dysfunctional mitochondria, thereby having an important role in homeostasis [64-66]. Although recent studies have examined the contribution of autophagy and mitophagy to the development of lung disorders, its pathogenetic role is not well established. Smoke exposure enhances autophagy in mouse lungs leading to emphysema, perhaps by increasing epithelial cell apoptosis [67, 68]. However, autophagy is supposedly specific to cell type and, thus, in alveolar macrophages in COPD patients, there appears to be a selective defect in autophagy resulting in impaired clearance of damaged and dysfunctional mitochondria [69]. After treatment with cigarette smoke, recent studies have shown altered mitochondrial structure (elongation) and function in alveolar epithelial cells [70], and in primary bronchial epithelial cells [71]. By contrast, HARA et al. [72] demonstrated mitochondrial fragmentation in primary human bronchial epithelial cells upon stimulation with cigarette smoke extract for $48 \mathrm{~h}$. It has been suggested that while mitochondria initially adapt to cigarette smoke and oxidative stress by altering their structure, this may be not beneficial in the long term due to a resultant decrease in mitophagy [70]. Recently, AHMAD et al. [73] proposed a pathway of smoke-induced cellular senescence due to impaired mitophagy. In 2012, Patel et al. [74] showed low levels of autophagy in IPF lungs. The suggested mechanism is TGF- $\beta$-induced activation of phosphatidylinositol-3 kinase and the mammalian target of rapamycin, which are known 
inhibitors of autophagy. It has been suggested that autophagy protects against lung fibrosis and, thus, smoking may suppress autophagy by inducing TGF- $\beta$ production. A more recent study by PATEL et al. [75] demonstrated damaged mitochondria in IPF lungs and mitochondrial depolarisation in TGF- $\beta$ treated lung epithelial cells. It had already been reported by BuENo et al. [76], in 2014, that a defect in mitophagy due to deficiency of a major regulatory molecule (PTEN-induced putative kinase (PINK)1) leads to mitochondrial damage and lung fibrosis.

\section{Is smoking a risk factor and does it affect the progression of ILDs?}

In IPF, smoking is now considered a risk factor, and probably interacts with genetic predisposition and other factors such as viral infections and gastro-oesophageal reflux [77]. Current or former smokers are almost $60 \%$ more likely than lifelong nonsmokers to develop IPF [78, 79]. In former smokers, the shorter the time interval from quitting smoking then the higher the likelihood of developing IPF is. Relatives of patients with familial IPF, which is either symptomatic or asymptomatic, with features of ILD on HRCT have a significantly higher prevalence of smoking compared with those without ILD features [80].

It should be stressed that, ideally, the exact effect of smoking on the development and progression of ILDs would be explored through longitudinal studies, which are very unlikely to ever be undertaken due to the relative rarity of ILDs and the high number of the scans required. However, recent studies of lung cancer screening and COPD may shed some light on this matter. In the COPD Gene study, $8 \%$ of smokers had interstitial lung abnormalities (ILAs) on HRCT, which were subclassified as subpleural, centrilobular, subpleural and centilobular, and "radiographic evidence of ILD". The presence of ILD was associated with a significantly higher likelihood of a history of smoking and significantly greater exposure to tobacco smoke in smokers [81]. A follow-up HRCT trial for cancer screening has identified ILAs in $9.7 \%$ of participants who were current or former smokers with a smoking history $>30$ pack-years [82]. Non-fibrotic ILAs, defined as ground-glass opacities, consolidations and mosaic attenuation, were present in $5.9 \%$ of cases whereas fibrotic ILAs, defined as reticulation with or without ground-glass opacities and honeycombing, were present in $2.1 \%$ of cases. After a follow-up of 2 years, $49 \%$ of non-fibrotic ILAs had improved and $11 \%$ had progressed. In contrast, none of the patients with fibrotic ILAs (which may represent UIP or fibrotic nonspecific interstitial pneumonia) had improved and 37\% had progressed [82]. However, the authors did not find any association between disease progression on HRCT and smoking status or smoking dose (pack-year smoking history). In another study, 25\% of smokers with initial abnormalities defined as "other chronic interstitial pneumonia" had progressed after 3 years, with progression always linked to continuation of smoking [83]. The question of whether smoking is the only risk factor responsible for the development of ILD in smokers remains unanswered. A panel of clinicians, radiologists and pathologists with expertise in ILDs were unable to accurately specify smoking status in a group of patients with an unknown smoking history simply by applying characteristic HRCT ILD features identified in a group of known smokers. For example, in one patient considered to have truly classical features of smoking-related ILD, there was no history of either active or passive smoking, providing indirect support for the contribution of other pathogenetic factors [84].

Surprisingly, it was suggested in one study that smoking may confer a protective effect against IPF progression, based on the observation that currently smoking IPF patients had a better survival [85]. However, this finding is likely to be related to the fact that smokers with more severe disease usually quit smoking whereas smokers with less severe disease are less likely to. Therefore, current smoking is associated with lengthier survival by virtue of linkage to less severe disease. The existence of this apparent paradox, a form of the "healthy smoker effect", was confirmed in a subsequent IPF study, in which current smokers lived longer but had no survival advantage after adjustment for initial disease severity using the composite physiologic index (which captures the functional effects of ILD while excluding those of emphysema [86]). In this study, survival was significantly better in never-smokers compared with former smokers and also with the combined group of former and current smokers [87].

The coexistence of emphysema and IPF has drawn attention recently. The entity, known as combined pulmonary fibrosis and emphysema (CPFE), is characterised by the presence, on HRCT, of emphysema mainly in the upper lobes and fibrosis mainly in the lower lobes although the two patterns can be also admixed (fig. 1) [88]. This entity has some peculiarities. First, there is a spurious preservation of lung volumes in association with a disproportionate and sometimes devastating reduction in diffusing capacity of the lung for carbon monoxide and transfer coefficient of the lung for carbon monoxide. This means that serial forced vital capacity (FVC), the most reliable means of detecting disease progression in ILD, is likely to be less accurate in this context. This is especially important in IPF where even a minimal reduction in FVC (i.e. 5-10\%) in 6 months has been associated with increased mortality [89]. These patients are more likely to develop pulmonary hypertension than IPF patients without emphysema with an important impact on survival [90], although it remains unclear whether a worse outcome merely reflects 
greater baseline disease severity when two separate disease processes are admixed. Importantly, it is sometimes difficult to discriminate between honeycombing and an admixture of emphysema and fibrosis on HRCT, a distinction that is pivotal in the diagnosis of IPF. CPFE can be also observed in the context of connective tissue disorders such as rheumatoid arthritis and systemic sclerosis [91, 92].

Clearly, smoking can indirectly affect the survival of patients with pulmonary fibrosis through the development of bronchogenic carcinoma. Certain intriguing similarities between lung cancer and IPF with regard to pathobiology and disease behaviour have been highlighted [93]. Conceivably, the development of lung cancer has a detrimental effect on survival of IPF patients. Recently, and in accordance with previous studies, it has been observed that patients with lung cancer have significantly lower median survival (38.7 months) than patients with IPF (63.9 months) [94]. This difference was mainly due to progression of lung cancer or to complications of IPF such as acute exacerbations after diagnostic or therapeutic procedures for lung cancer. Patients in the early stage of both diseases have better survival and a lower likelihood of complications than patients with severe disease. Therefore, early diagnosis and the accurate staging of severity in both diseases are equally important.

\section{Conclusion}

Our knowledge regarding the involvement of smoking in the pathogenesis of ILDs has evolved in the recent years. Smoking is now considered the principal pathogenetic factor for RB-ILD, DIP and PLCH, whereas it is an important cofactor, probably acting in synergy with genetic and environmental factors, for the development of other ILDs such as IPF and RA-ILD. It definitely has a significant impact on the survival of patients with either an isolated ILD or in cases of concurrent development of emphysema and lung cancer, two well established smoking-related lung diseases. Therefore, smoking cessation should be advised in all patients with ILDs.

\section{References}

1 Houghton M, Mouded M, Shapiro SD. Common origins of lung cancer and COPD. Nat Med 2008; 14: 1023-1024.

2 American Thoracic Society. Cigarette smoking and health. Am J Respir Crit Care Med 1996; 153: 861-865.

3 Murin S, Hilbert J, Reilly SJ. Cigarette smoking and the lung. Clin Rev Allergy Immunol 1997; 15: 307-361.

4 Vassallo R, Ryu JH. Smoking-related interstitial lung diseases. Clin Chest Med 2012; 33: 165-178.

5 Caminati A, Cavazza A, Sverzellati N, et al. An integrated approach in the diagnosis of smoking-related interstitial lung diseases. Eur Respir Rev 2012; 21: 207-217.

6 Wells AU, Nicholson AG, Hansell DM. Challenges in pulmonary fibrosis. 4: smoking-induced diffuse interstitial lung diseases. Thorax 2007; 62: 904-910.

$7 \quad$ Peros-Golubicic T, Ljubic S. Cigarette smoking and sarcoidosis. Acta Med Croatica 1995; 49: $187-193$.

8 Sieminska A, Kuziemski K. Respiratory bronchiolitis-interstitial lung disease. Orphanet J Rare Dis 2014 ; 9: 106.

9 D'hulst AI, Vermaelen KY, Brusselle GG, et al. Time course of cigarette smoke-induced pulmonary inflammation in mice. Eur Respir J 2005; 26: 204-213.

10 Niewoehner DE, Kleinerman J, Rice DB. Pathologic changes in the peripheral airways of young cigarette smokers. N Engl J Med 1974; 291: 755-758.

11 Remy-Jardin M, Remy J, Gosselin B, et al. Lung parenchymal changes secondary to cigarette smoking: pathologic-CT correlations. Radiology 1993; 186: 643-651.

12 Fraig M, Shreesha U, Savici D, et al. Respiratory bronchiolitis: a clinicopathologic study in current smokers, ex-smokers, and never-smokers. Am J Surg Pathol 2002; 26: 647-653.

13 Moon J, du Bois RM, Colby TV, et al. Clinical significance of respiratory bronchiolitis on open lung biopsy and its relationship to smoking related interstitial lung disease. Thorax 1999; 54: 1009-1014.

14 Rahman S, Biswas K, Kode A. Oxidant and antioxidant balance in the airways and airway diseases. Eur Pharmacol 2006; 533: 222-239.

15 Van der Vaart H, Postma DS, Timens W, et al. Acute effects of cigarette smoke on inflammation and oxidative stress: a review. Thorax 2004; 59: 713-721.

16 Zavitz CC, Gaschler GJ, Robbins CS, et al. Impact of cigarette smoke on $\mathrm{T}$ and $\mathrm{B}$ cell responsiveness. Cell Immunol 2008; 253: 38-44.

17 Rennard SI, Togo S, Holz O. Cigarette smoke inhibits alveolar repair: a mechanism for the development of emphysema. Proc Am Thorac Soc 2006; 3: 703-708.

18 Kuschner WG, D'Alessandro A, Wong $\mathrm{H}$, et al. Dose-dependent cigarette smoking-related inflammatory responses in healthy adults. Eur Respir J 1996; 9: 1989-1994.

19 Arnson Y, Shoenfeld Y, Amital H. Effects of tobacco smoke on immunity, inflammation and autoimmunity. J Autoimmun 2010; 34: J258-J265.

20 Lu LM, Zavitz CC, Chen B, et al. Cigarette smoke impairs NK cell-dependent tumor immune surveillance. J Immunol 2007; 178: 936-943.

21 Kode A, Yang SR, Rahman I. Differential effects of cigarette smoke on oxidative stress and proinflammatory cytokine release in primary human airway epithelial cells and in a variety of transformed alveolar epithelial cells. Respir Res 2006; 7: 132.

22 Vassallo R, Tamada K, Lau JS, et al. Cigarette smoke extract suppresses human dendritic cell function leading to preferential induction of Th-2 priming. J Immunol 2005; 175: 2684-2691.

23 Israel-Assayag E, Dakhama A, Lavigne S, et al. Expression of costimulatory molecules on alveolar macrophages in hypersensitivity pneumonitis. Am J Respir Crit Care Med 1999; 159: 1830-1834. 
2011; 2011: 461439.

40 Fischer A, Solomon JJ, du Bois RM, et al. Lung disease with anti-CCP antibodies but not rheumatoid arthritis or connective tissue disease. Respir Med 2012; 106: 1040-1047.

41 Klareskog L, Stolt P, Lundberg K, et al. A new model for an etiology of rheumatoid arthritis: smoking may trigger HLA-DR (shared epitope)-restricted immune reactions to autoantigens modified by citrullination. Arthritis Rheum 2006; 54: 38-46.

42 Harlow L, Rosas IO, Gochuico BR, et al. Identification of citrullinated hsp90 isoforms as novel autoantigens in rheumatoid arthritis-associated interstitial lung disease. Arthritis Rheum 2013; 65: 869-879.

43 Giles JT, Danoff SK, Sokolove J, et al. Association of fine specificity and repertoire expansion of anticitrullinated peptide antibodies with rheumatoid arthritis associated interstitial lung disease. Ann Rheum Dis 2014; 73: 1487-1494.

44 Antoniou KM, Margaritopoulos GA, Tomassetti S, et al. Interstitial lung disease. Eur Respir Rev 2014; 23: 40-54. pulmonary fibrosis. Am J Respir Crit Care Med 2012; 186: 306-313.

46 Morse D, Rosas IO. Tobacco smoke-induced lung fibrosis and emphysema. Annu Rev Physiol 2014; 76: $493-513$.

47 Collard HR. The age of idiopathic pulmonary fibrosis. Am J Respir Crit Care Med 2010; 181: 771-772.

48 Chilosi M, Poletti V, Rossi A. The pathogenesis of COPD and IPF: distinct horns of the same devil? Respir Res 2012; 13: 3 .

49 López-Otín C, Blasco MA, Partridge L, et al. The hallmarks of aging. Cell 2013; 153: 1194-1217.

50 Borie R, Crestani B, Bichat H. Prevalence of telomere shortening in familial and sporadic pulmonary fibrosis is increased in men. Am J Respir Crit Care Med 2009; 179: 1073.

51 Armanios M. Telomerase and idiopathic pulmonary fibrosis. Mutat Res 2012; 730: 52-58.

52 Armanios MY, Chen JJ, Cogan JD, et al. Telomerase mutations in families with idiopathic pulmonary fibrosis. N Engl J Med 2007; 356: 1317-1326.

53 Chan SR, Blackburn EH. Telomeres and telomerase. Philos Trans R Soc Lond B Biol Sci 2004; 359: 109-121.

54 Valdes AM, Andrew T, Gardner JP, et al. Obesity, cigarette smoking, and telomere length in women. Lancet 2005; 366: 662-664.

55 DeMarini DM. Genotoxicity of tobacco smoke and tobacco smoke condensate: a review. Mutat Res 2004; 567: 447-474.

56 Brack AS, Conboy MJ, Roy S, et al. Increased Wnt signaling during aging alters muscle stem cell fate and increases fibrosis. Science 2007; 317: 807-810.

57 Jones DP. Extracellular redox state: refining the definition of oxidative stress in aging. Rejuvenation Res 2006; 9: $169-181$.

58 Ryter SW, Lee SJ, Choi AMK. Autophagy in cigarette smoke-induced chronic obstructive pulmonary disease. Expert Rev Respir Med 2010; 4: 573-584.

59 Cuervo AM. Autophagy and aging: keeping that old broom working. Trends Genet 2008; 24 : 604-612.

60 Choi AM, Ryter SW, Levine B. Autophagy in human health and disease. N Engl J Med 2013; 368: 651-662.

61 Deretic V, Saitoh T, Akira S. Autophagy in infection, inflammation and immunity. Nat Rev Immunol 2013; 13: $722-737$.

62 Nakahira K, Choi AM. Autophagy: a potential therapeutic target in lung diseases. Am J Physiol Lung Cell Mol Physiol 2013; 305: L93-L107.

63 Margaritopoulos GA, Tsitoura E, Tzanakis N, et al. Self-eating: friend or foe? The emerging role of autophagy in idiopathic pulmonary fibrosis. Biomed Res Int 2013; 2013: 420497. 
64 Wang $\mathrm{CH}, \mathrm{Wu} \mathrm{SB}, \mathrm{Wu} \mathrm{YT}$, et al. Oxidative stress response elicited by mitochondrial dysfunction: implication in the pathophysiology of aging. Exp Biol Med (Maywood) 2013; 238: 450-460.

65 Palikaras K, Tavernarakis N. Mitochondrial homeostasis: the interplay between mitophagy and mitochondrial biogenesis. Exp Gerontol 2014; 56: 182-188.

66 Sureshbabu A, Bhandari V. Targeting mitochondrial dysfunction in lung diseases: emphasis on mitophagy. Front Physiol 2013; 4: 384.

67 Chen ZH, Kim HP, Sciurba FC, et al. Egr-1 regulates autophagy in cigarette smoke-induced chronic obstructive pulmonary disease. PLoS One 2008; 3: e3316.

68 Chen ZH, Lam HC, Jin Y, et al. Autophagy protein microtubule-associated protein 1 light chain-3B (LC3B) activates extrinsic apoptosis during cigarette smoke-induced emphysema. Proc Natl Acad Sci USA 2010; 107: $18880-18885$.

69 Monick MM, Powers LS, Walters K, et al. Identification of an autophagy defect in smokers' alveolar macrophages. J Immunol 2010; 185: 5425-5435.

70 Ballweg K, Mutze K, Königshoff $\mathrm{M}$, et al. Cigarette smoke extract affects mitochondrial function in alveolar epithelial cells. Am J Physiol Lung Cell Mol Physiol 2014; 307: L895-L907.

71 Hoffmann RF, Zarrintan S, Brandenburg SM, et al. Prolonged cigarette smoke exposure alters mitochondrial structure and function in airway epithelial cells. Respir Res 2013; 14: 97.

72 Hara H, Araya J, Ito S, et al. Mitochondrial fragmentation in cigarette smoke-induced bronchial epithelial cell senescence. Am J Physiol Lung Cell Mol Physiol 2013; 305: L737-L746.

73 Ahmad T, Sundar IK, Lerner CA, et al. Impaired mitophagy leads to cigarette smoke stress-induced cellular senescence: implications for chronic obstructive pulmonary disease. FASEB J 2015; 29: 2912-2929.

74 Patel AS, Lin L, Geyer A, et al. Autophagy in idiopathic pulmonary fibrosis. PLoS One 2012; 7: e41394.

75 Patel AS, Song JW, Chu SG, et al. Epithelial cell mitochondrial dysfunction and PINK1 are induced by transforming growth factor-betal in pulmonary fibrosis. PLoS One 2015; 10: e0121246.

76 Bueno M, Lai YC, Romero Y, et al. PINK1 deficiency impairs mitochondrial homeostasis and promotes lung fibrosis. J Clin Invest 2015; 125: 521-538.

77 Margaritopoulos GA, Giannarakis I, Siafakas NM, et al. An update on idiopathic pulmonary fibrosis. Panminerva Med 2013; 55: 109-120.

78 Hubbard R, Lewis S, Richards K, et al. Occupational exposure to metal or wood dust and aetiology of cryptogenic fibrosing alveolitis. Lancet 1996; 347: 284-289.

79 Baumgartner KB, Samet JM, Stidley CA, et al. Cigarette smoking: a risk factor for idiopathic pulmonary fibrosis. Am J Respir Crit Care Med 1997; 155: 242-248.

80 Rosas IO, Ren P, Avila NA, et al. Early interstitial lung disease in familial pulmonary fibrosis. Am J Respir Crit Care Med 2007; 176: 698-705.

81 Washko GR, Hunninghake GM, Fernandez IE, et al. Lung volumes and emphysema in smokers with interstitial lung abnormalities. N Engl J Med 2011; 364: 897-906.

82 Jin GY, Lynch D, Chawla A, et al. Interstitial lung abnormalities in a CT lung cancer screening population: prevalence and progression rate. Radiology 2013; 268: 563-571.

83 Sverzellati N, Guerci L, Giorgia R, et al. Interstitial lung diseases in a lung cancer screening trial. Eur Respir J 2011; 38: 392-400

84 Flaherty KR, Fell C, Aubry MC, et al. Smoking-related idiopathic interstitial pneumonia. Eur Respir J 2014; 44: 594-602.

85 King TE Jr, Schwarz MI, Brown K, et al. Idiopathic pulmonary fibrosis: relationship between histopathologic features and mortality. Am J Respir Crit Care Med 2001; 164: 1025-1032.

86 Wells AU, Desai SR, Rubens MB, et al. Idiopathic pulmonary fibrosis: a composite physiologic index derived from disease extent observed by computed tomography. Am J Respir Crit Care Med 2003; 167: 962-969.

87 Antoniou KM, Hansell DM, Rubens MB, et al. Idiopathic pulmonary fibrosis: outcome in relation to smoking status. Am J Respir Crit Care Med 2008; 177: 190-194.

88 Cottin V, Nunes H, Brillet PY, et al. Combined pulmonary fibrosis and emphysema: a distinct underrecognised entity. Eur Respir J 2005; 26: 586-593.

89 Zappala CJ, Latsi PI, Nicholson AG, et al. Marginal decline in forced vital capacity is associated with a poor outcome in idiopathic pulmonary fibrosis. Eur Respir J 2010; 35: 830-836.

90 Cottin V, Le Pavec J, Prevot G, et al. Pulmonary hypertension in patients with combined pulmonary fibrosis and emphysema syndrome. Eur Respir J 2010; 35: 105-111.

91 Antoniou KM, Walsh SL, Hansell DM, et al. Smoking-related emphysema is associated with idiopathic pulmonary fibrosis and rheumatoid lung. Respirology 2013; 18: 1191-1196.

92 Cottin V, Nunes H, Mouthon L, et al. Combined pulmonary fibrosis and emphysema syndrome in connective tissue disease. Arthritis Rheum 2011; 63: 295-304.

93 Vancheri C, Failla M, Crimi N, et al. Idiopathic pulmonary fibrosis: a disease with similarities and links to cancer biology. Eur Respir J 2010; 35: 496-504.

94 Tomassetti S, Gurioli C, Ryu JH, et al. The impact of lung cancer on survival of idiopathic pulmonary fibrosis. Chest 2015; 147: 157-164. 